\title{
$\mathbb{B} \Delta+$ COMMUNICATIONS MATERIALS
}

ARTICLE

Check for updates

https://doi.org/10.1038/s43246-020-00078-y OPEN

\section{Nacre toughening due to cooperative plastic deformation of stacks of co-oriented aragonite platelets}

Hyun-Chae Loh (10) 1, Thibaut Divoux (10) 1,2,3, Bernd Gludovatz (10) 4, Pupa U. P. A. Gilbert (1) 5,6,7, Robert O. Ritchie (iD) ${ }^{8}$, Franz-Josef Ulm ${ }^{1,2} \&$ Admir Masic (iD) ${ }^{1 凶}$

Nacre's structure-property relationships have been a source of inspiration for designing advanced functional materials with both high strength and toughness. These outstanding mechanical properties have been mostly attributed to the interplay between aragonite platelets and organic matrices in the typical brick-and-mortar structure. Here, we show that crystallographically co-oriented stacks of aragonite platelets, in both columnar and sheet nacre, define another hierarchical level that contributes to the toughening of nacre. By correlating piezo-Raman and micro-indentation results, we quantify the residual strain energy associated with strain hardening capacity. Our findings suggest that the aragonite stacks, with characteristic dimensions of around $20 \mu \mathrm{m}$, effectively store energy through cooperative plastic deformation. The existence of a larger length scale beyond the brick-and-mortar structure offers an opportunity for a more efficient implementation of biomimetic design.

\footnotetext{
${ }^{1}$ Department of Civil and Environmental Engineering, Massachusetts Institute of Technology, 77 Massachusetts Avenue, Cambridge, MA 02139, USA ${ }^{2}$ MultiScale Material Science for Energy and Environment, UMI 3466, CNRS-MIT, 77 Massachusetts Avenue, Cambridge, MA 02139, USA. ${ }^{3}$ Univ Lyon, Ens de Lyon, Univ Claude Bernard, CNRS, Laboratoire de Physique, F-69342 Lyon, France. ${ }^{4}$ School of Mechanical and Manufacturing Engineering, UNSW Sydney, Sydney, NSW 2052, Australia. ${ }^{5}$ Department of Physics, University of Wisconsin, Madison, WI 53706, USA. ${ }^{6}$ Departments of Chemistry, Geoscience, Materials Science and Engineering, University of Wisconsin, Madison, WI 53706, USA. ${ }^{7}$ Lawrence Berkeley National Laboratory, Berkeley, CA 94720, USA. ${ }^{8}$ Department of Materials Science and Engineering, University of California, Berkeley, CA 94720, USA.

$凶_{\text {email: }}$ asic@mit.edu
} 
O rganisms have evolved over geologic time to fine-tune their structural materials with multi-functionality and remarkable mechanical properties ${ }^{1,2}$. Inspired by nature, scientists and engineers have mimicked some of these materials to design advanced functional materials ${ }^{3,4}$. Most of the structural materials found in nature are characterized by multi-scale hierarchy, which confers them multiple functions and mechanical benefits ${ }^{5}$. Among biological materials, mollusk shells have been extensively studied for their outstanding combination of stiffness, strength, and toughness ${ }^{6-9}$. The shell is a composite, primarily made of two different polymorphs of calcium carbonate: calcite and aragonite. In many shells, the outer layer is composed of calcite prisms and the inner layer-nacre-has a "brick-andmortar" structure, where $\sim 500 \mathrm{~nm}$ thick aragonite $\left(\mathrm{CaCO}_{3}\right)$ platelets (>95\% of mass) are interspersed with an organic matrix, mainly composed of elastic biopolymers, such as chitin and proteins ${ }^{9}$. Each platelet behaves as a single crystal ${ }^{10}$, and only small misorientation was found owing to nanoparticles and twinning domains ${ }^{11-13}$.

Nacre's work of fracture is 3000 times that of monolithic aragonite ${ }^{7}$, and its high toughness has been attributed to various chemo-mechanical mechanisms generally related to its hier-

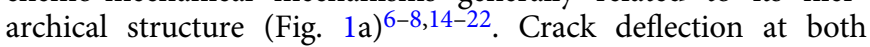
micro- and mesoscales, crack blunting, and microcrack formation all contribute to enhanced toughness by creating tortuous cracks of a limited extent and preventing catastrophic failures ${ }^{6-8,14}$. Although only a tiny portion $(<5 \%)$ of the material, the organic matrix serves greatly to toughen the nacre by dissipating energy as cracks open through bridging between the platelets and unfolding its protein structure $8,15,16$. Moreover, nano-asperities found on platelet interfaces and platelet pull-out are important mechanisms that increase the shear strength and tensile toughness of nacre ${ }^{17-21}$. Finally, the mineral bridges between the platelets increase toughness, shear strength, and stiffness ${ }^{20,22}$.

Over the past two decades, structure-function relationships of complex structural materials, including nacre, have been analyzed using stress and strain-mapping techniques, such as contour mapping, computed tomography, digital image correlation, electron backscatter diffraction, neutron diffraction, and synchrotron X-ray diffraction ${ }^{23-31}$. In addition to these approaches, Raman spectroscopy is a powerful chemo-mechanical characterization method that allows stress and strain mapping through a technique known as piezo-Raman ${ }^{32-38}$. Stress-induced changes in the crystal lattice and molecular vibrational modes are reflected in a linear relationship between the external/residual stress and Raman peak position. To date, piezo-Raman provided essential links between chemical composition and mechanical properties in materials such as silicon ${ }^{28,39-42}$, diamond ${ }^{39}$, graphene ${ }^{43}$, and hydroxyapatite ${ }^{35,36}$.

Tensile or flexural bending tests have been used to study the tensile behavior of nacre at both macroscopic and submicron scales $^{6-8,14,17,18}$. However, the quantitative analysis of the crack size is difficult in these experiments, and therefore, comparing the mechanical properties with the structure's length scale is challenging. Previously, larger cracks were differentiated from natural platelet boundaries by comparing the dilation of the interfaces ${ }^{18}$; however, it remains challenging to clearly differentiate smaller cracks and crack tips from natural boundaries. Micro- and nanoindentation are well-established mechanical testing methods to indirectly measure the material's tensile properties, where the tensile load is generated due to the wedge-like shape of the indenter ${ }^{44}$. Compared to tensile or flexural bending tests, indentation methods are advantageous when studying the
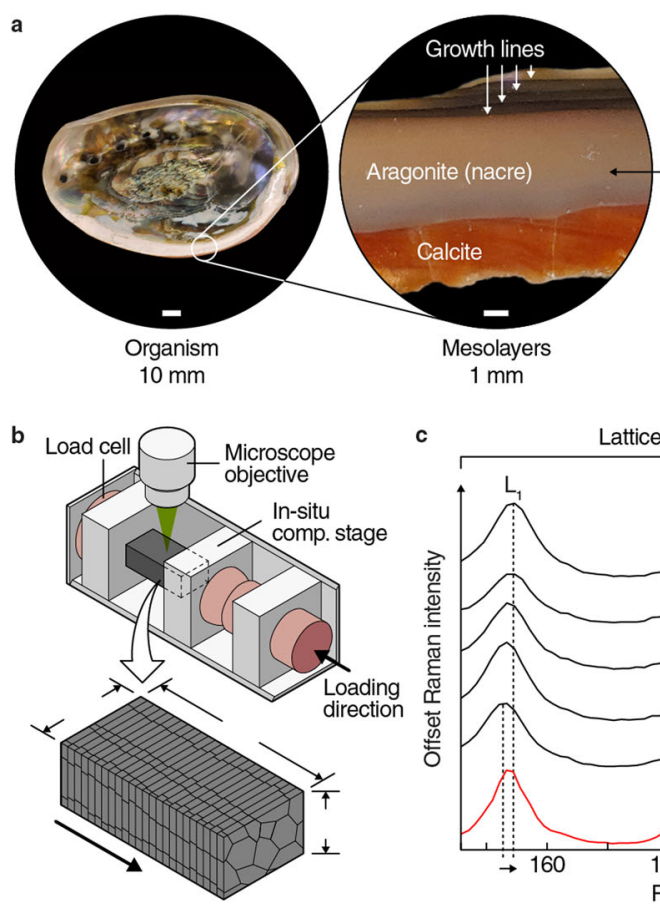

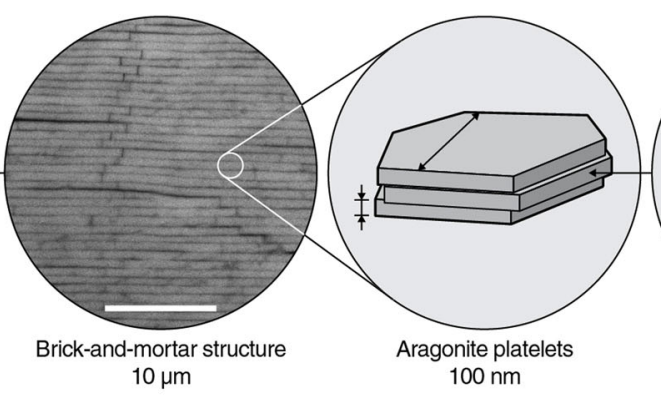

$100 \mathrm{~nm}$

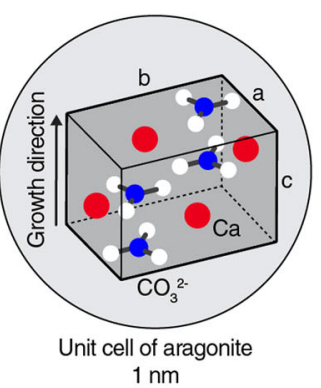

d

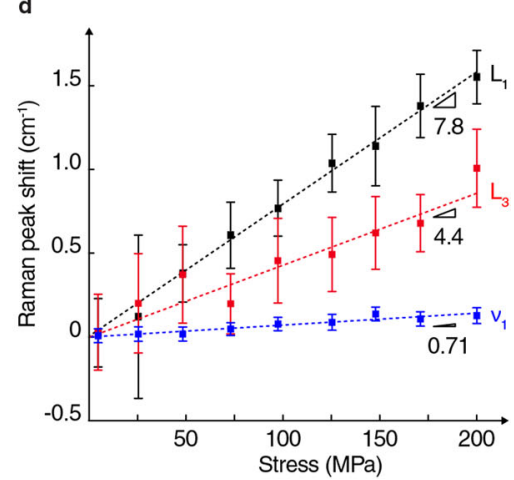

Fig. 1 The hierarchical structure of an abalone shell and its piezo-Raman spectroscopy. a The hierarchical structure of an abalone (Haliotis rufescens) shell. Scale bars are $10 \mathrm{~mm}$ (organism scale), $1 \mathrm{~mm}$ (mesolayer scale), and $10 \mu \mathrm{m}$ (brick-and-mortar structure). b Schematics of the compression stage designed for collecting Raman data during compression tests. The aragonite platelets are illustrated on the sample to visualize the orientation of the sample. Aragonite platelets are not in scale. c Average Raman spectra of nacre sample over $10 \times 10 \mu \mathrm{m}^{2}$ during uniaxial compression tests under various loads ranging between $0 \mathrm{MPa}$ and $200 \mathrm{MPa}$. d Piezo-Raman relationship of nacre; the data points and the error bars indicate the average and the standard deviation of the peak positions, respectively. 
size-effect and fractographic patterns since the dimensions can be easily quantified by controlling the indent size ${ }^{45}$.

Despite numerous studies on nacre toughening mechanisms at both macroscopic and submicron scales ${ }^{6-17}$, the potential mechanical contribution of aragonite stacks (i.e., crystallographically co-oriented stacks of platelets; $10-100 \mu \mathrm{m}$ scale) ${ }^{10,46-50}$, have yet to be explored. A hierarchical structure ("supertablet") with a characteristic length of about $10 \mu \mathrm{m}$ was found to contribute to the material's toughness, but the structural characteristics that distinguish each supertablet were not identified ${ }^{21}$. Here, we combine piezo-Raman and microindentation to quantify the residual strain energy, which is associated with the strain hardening capacity of nacre. Moreover, we compare the micro-indentation results with those from macroscopic mechanical tests to confirm the crack propagation pattern. From the results, we show that the cooperative movement of the co-oriented aragonite stacks contributes to the strain hardening of the nacre, defining a new length scale of nacre toughening.

\section{Results and discussion}

Piezo-Raman relationships in nacre. A Raman spectrum is composed of a series of peaks associated with molecular vibration modes. Therefore, each material's Raman spectrum can serve to identify its different components. Moreover, a material's Raman peak width, peak shift, and polarization sensitivity can be related to its crystallinity, chemo-mechanical environment, and crystal orientation, respectively ${ }^{29}$. In this context, minute changes in peak features can be monitored in the process of analyzing the structural and chemo-mechanical properties of nacre in a spatially resolved fashion (lateral resolution $\sim 0.3 \mu \mathrm{m}$ and depth resolution $\sim 3 \mu \mathrm{m})^{29}$.

According to group theory, aragonite has 30 Raman active peaks $^{51}$. However, in practice, not all the theoretical peaks are detectable due to limitations in spectral resolution and peak intensities. The Raman spectrum of aragonite is presented on the lower part of Fig. 1c, where the strongest peaks, highlighted by vertical dashed lines, are assigned using the theoretical predictions of the peak positions $s^{51}$. In the low energy region of the spectrum, the scattering signals correspond to the lattice vibration modes $\left(\mathrm{L}_{1}\right.$, $\mathrm{L}_{2}$, and $\left.\mathrm{L}_{3}\right)$. In contrast, the most intense peak at $1085 \mathrm{~cm}^{-1}$ corresponds to the symmetric stretching mode $\left(v_{1}\right)$ of the carbonate ion $\left(\mathrm{CO}_{3}{ }^{2-}\right)$. The peaks $\mathrm{L}_{1}\left(\sim 152 \mathrm{~cm}^{-1}\right), \mathrm{L}_{3}\left(\sim 206 \mathrm{~cm}^{-1}\right)$, and $v_{1}\left(\sim 1085 \mathrm{~cm}^{-1}\right)$ are associated with the $\mathrm{B}_{1 \mathrm{~g}}, \mathrm{~B}_{2 \mathrm{~g}}$, and $\mathrm{A}_{\mathrm{g}}$ symmetries, respectively ${ }^{51}$, and the Raman tensors associated with these vibrational modes are listed in Supplementary Table 1.

The piezo-Raman relationships of nacre were determined by measuring the Raman peak shifts at different stress levels using an in-situ uniaxial compression test (Fig. 1b). Nacre's Raman spectra under uniaxial compression are illustrated in Fig. $1 \mathrm{c}$ at various levels of compressive stress (up to $200 \mathrm{MPa}$ ) and compared to the spectrum of geologic monolithic aragonite. We observe that the Raman shift of the peaks $\mathrm{L}_{1}, \mathrm{~L}_{3}$, and $v_{1}$ scales linearly with the applied stress (Fig. 1d), and the piezo-Raman relationships $v(\sigma)=v_{0}+r \cdot \sigma$ can be obtained by the least-square fitting of the experimental data (Supplementary Table 1), where $v_{0}\left[\mathrm{~cm}^{-1}\right]$ is the wavenumber of the Raman peak in the ambient condition, and $r\left[\mathrm{~cm}^{-1}\right.$ per $\left.\mathrm{GPa}\right]$ is the piezo-Raman coefficient. Interestingly, the peaks with lower scattering energy are more sensitive to the applied stress, leading to larger piezo-Raman coefficients. For this reason, we used the peak $\mathrm{L}_{1}$ for computing the residual stress induced by micro-indentation tests on nacre samples, which resulted in better signal-to-noise ratios and a better stress assessment.

Residual stress and energy analysis on indented samples. We combined piezo-Raman with micro-indentation to explore the mechanical behaviors of monolithic aragonite and nacre (see "Methods" section). The indentation (maximum load of $0.8 \mathrm{~N}$ ) of a monolithic aragonite sample generates a large crack (right side of Fig. 2a). The corresponding Raman $\mathrm{L}_{1}$ peak shift map (Fig. 2b) and the computed residual stress determined with the piezoRaman relationship (Fig. 2c) of the indented area show that the stress remains confined within the indented area. In contrast, the same test performed on a nacre sample reveals a strikingly different scenario (Fig. 2d-f). The SEM image in Fig. 2d does not display any major crack, indicating that the damage is confined to the vicinity of the indent. Moreover, the Raman peak shift is observed over a large area of about $20 h_{\max }$, where $h_{\max }$ denotes the maximum penetration depth reached by the indenter during the test (Fig. 2e). The computed residual stress (Fig. 2f) shows an anisotropic, butterfly-like pattern, where the main axis coincides with the growth direction of the platelet $(y$-axis denoted in Fig. 2e). Finally, the "lobes" along the $x$ - and $y$-axes show tensile and compressive residual stress, respectively (see Supplementary Fig. 1 for the peak shift profile along the $x$ - and $y$-axes). These observations are robust and hold when increasing the maximum load of the indentation test. Some cracks appear in the sample for larger loads. However, they remain confined in the vicinity of the indented area (see, for instance, the result of an indentation test with a maximum load of $5 \mathrm{~N}$ in Fig. $2 \mathrm{~g}$ ), and the peak position and residual stress analyses show similar distributions as those of the smaller indentation load (Fig. 2h, i). The spatial extent of the pattern is larger for increasing indentation loads, and the results are consistent for tests performed on different nacre samples for various levels of stress (Supplementary Fig. 2). Finally, the pattern was consistent regardless of the orientation of the Berkovitch indenter relative to the sample, indicating that the stress distribution is influenced by the anisotropy in crystallographic texture (i.e., brick-and-mortar structure), and not by the geometric anisotropy of the indenter (Supplementary Fig. 3).

We now quantify and compare the work provided during the force-driven indentation test with the residual strain energy estimated using piezo-Raman, for a broad range of indentation tests performed to various maximum depths. The goal of comparing these two quantities is to evaluate the energy storage capacity of nacre's microstructure. The energy storage capacity is defined as the ability to store externally supplied work (here provided by the indentation test) as residual energy. In their natural environment during the life of the animal, shells are exposed to repeated impacts from debris and predators. Loading and unloading on the surface of the material generate residual stress when the load exceeds the elastic limit ${ }^{52}$. This local residual stress prevents the material from yielding when the next load is applied and acts as a protective layer that hardens the material ${ }^{52}$. Therefore, the greater the residual energy stored into the microstructure, the greater the plastic hardening capacity of the material.

First, we calculated the input work IW $=\int_{0}^{h_{\max }} P(h) d h$ from the integral of the force-indentation depth curve $P(h)$, where $P$ is the indentation force, and $h$ is the indentation depth (see Supplementary Fig. 4). Second, we use the piezo-Raman relationship for determining the hardening or frozen energy after unloading. The residual stresses in the material bulk inferred from piezo-Raman thus can be used for estimating the residual strain energy (RSE) by integrating the residual strain energy density, $u_{\text {res }}$, after noise subtraction (Supplementary Note 1) over the volume affected by the indentation test:

$$
\mathrm{RSE}=\iiint_{V} u_{\mathrm{res}} d V=\int_{0}^{z_{\max }} E_{\mathrm{res}}(z) d z
$$

where $E_{\text {res }}(z)=e-e_{\text {noise }}$ is the noise corrected lineic RSE at depth $z$, and $z_{\max }$ denotes the depth of the indentation interaction 

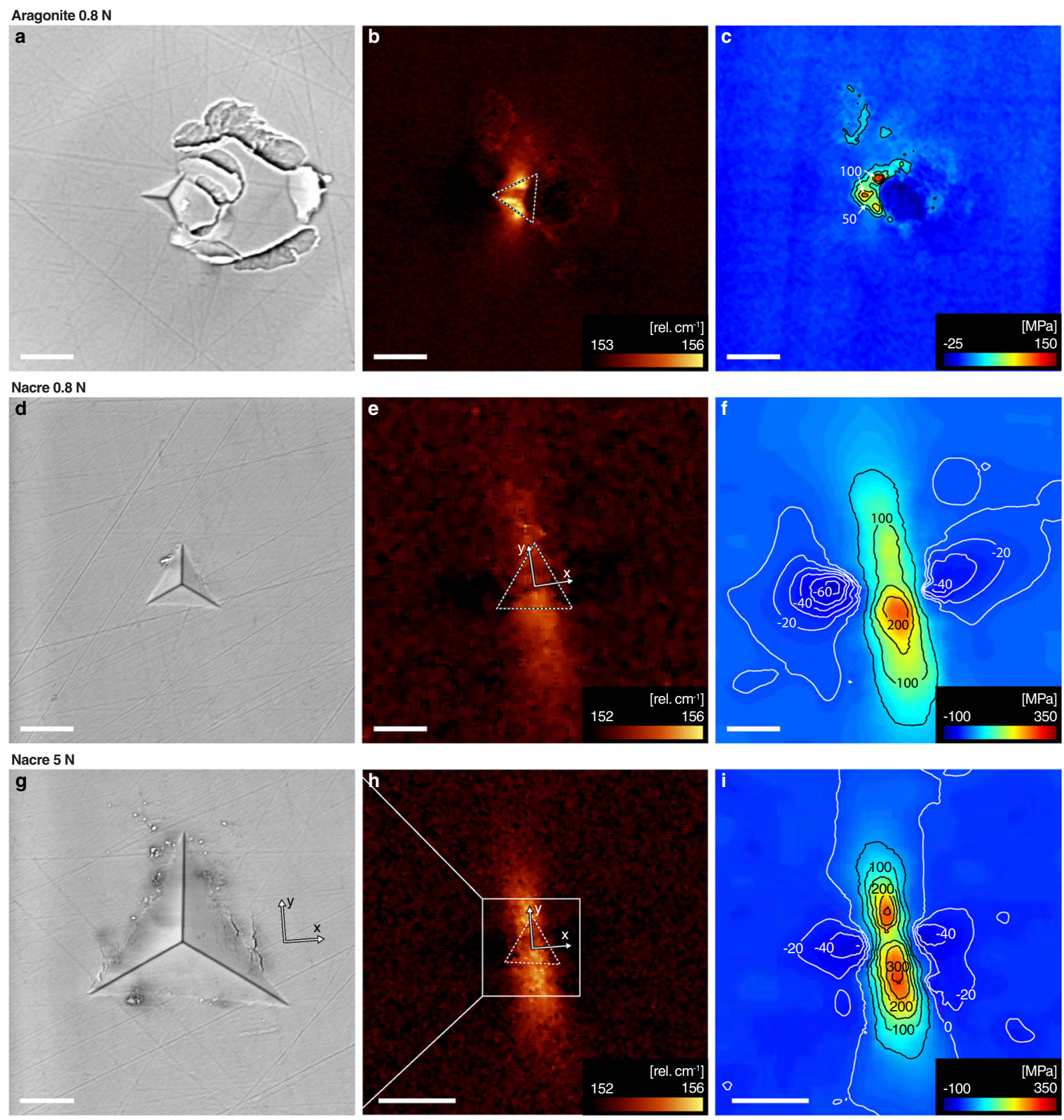

Fig. 2 Residual stress maps show different stress distribution in brittle (aragonite) and ductile materials (nacre). Each row displays SEM observation, Raman $L_{1}$ peak shift map, and stress analyses on the same region of a micro-indented $\mathbf{a}-\mathbf{c}$ monolithic aragonite (maximum load $\left.0.8 \mathrm{~N}\right), \mathbf{d}-\mathbf{f}$ nacre $(0.8 \mathrm{~N})$, and $\mathbf{g}$-i nacre $(5 \mathrm{~N})$. a SEM image of the indent; large cracks originate from the edges of the indent. $\mathbf{b}$ A false-color heatmap of the $L_{1}$ peak shift with the color corresponding to the peak position (153-156 rel cm${ }^{-1}$ ); the indent position is marked with a dashed line. c Contour and false-color heatmap of the residual stress computed with the piezo-Raman relationship. $\mathbf{d}, \mathbf{g}$ SEM images of the indents on nacre show minimal crack propagation. The aragonite platelet width direction ( $x$-axis) and the growth direction ( $y$-axis) are marked with arrows. $\mathbf{e}$, $\mathbf{h}$ Raman peak position maps illustrate that the peak shift is larger along the growth direction. Color scale bars are 152-156 rel cm${ }^{-1}$. f, $\mathbf{i}$ The iso-stress contours and the heatmap show the anisotropic distribution of the residual stress along the growth direction. The scale bars are $20 \mu \mathrm{m}$ in $(\mathbf{a}-\mathbf{g})$ and $100 \mu \mathrm{m}$ in $(\mathbf{h}-\mathbf{i})$.

volume. The interaction volume is defined as the material volume sensed in an indentation test. The lineic energy $e=\iint_{A} u d A$ was estimated from the strain energy density, $u=\sigma^{2} / 2 E$, where $\sigma$ is the residual stress inferred from piezo-Raman, and $E$ is the elastic modulus determined from the indentation test ${ }^{53}$. The determination procedure to obtain $E_{\text {res }}(z)$ is sketched in Supplementary Fig. 5, and details are provided in Supplementary Note 1.

It is noteworthy in our approach to determining RSE that aragonite is a translucent to transparent crystal, which allows us to measure Raman spectra from different focal planes inside the material using a confocal Raman micro-spectroscopy system. As an illustration, Fig. 3 a shows a $3 \mathrm{D}$ stress distribution reconstructed from a series of 2D Raman scans obtained from different focal depths (see Supplementary Movie 1 and Supplementary Fig. 6 for animated visualization and 2D snapshots of the 3D stress field). Finally, we computed the RSE by integrating $E_{\text {res }}(z)$ over the affected depth (the area left of the curve in Fig. 3b). We observed a similar stress distribution for every indent scanned in 3D, which allowed us to estimate the RSE from 2D piezo-Raman scans on all other indents.

IW and RSE were calculated for 58 independent indents performed with different loads, and the results are reported in Fig. $3 \mathrm{c}$ as a function of the characteristic size of indentation interaction volume $\left(d=3 h_{\max }\right)$, estimated to be three times the 
a

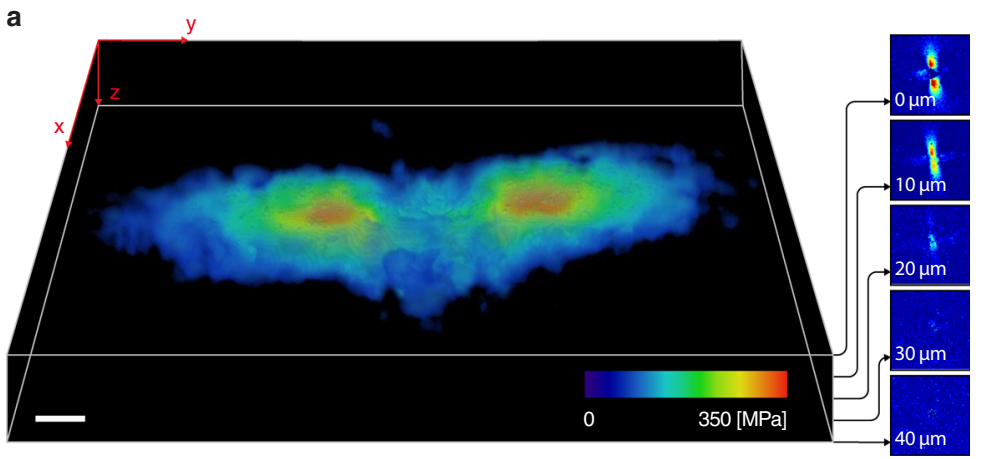

b Lineic residual strain energy $E_{\text {( }}(\mathrm{mJ} / \mathrm{m})$

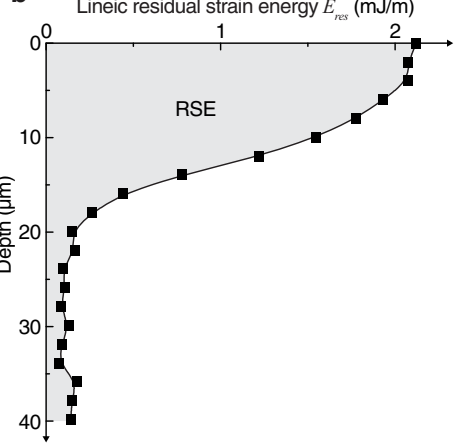

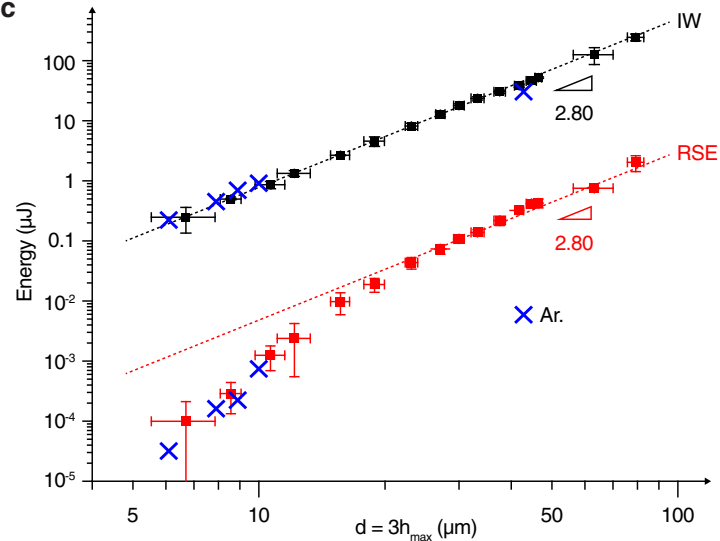

d

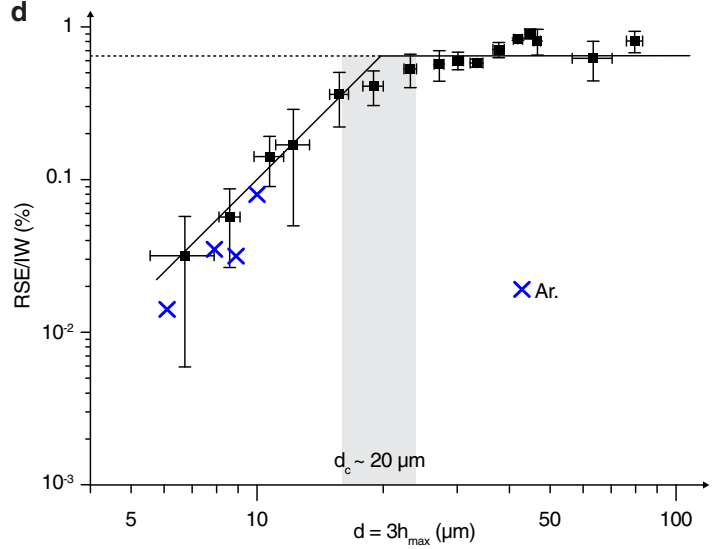

Fig. 3 Strain hardening mechanism is activated at a particular length scale. a $A$ perspective $3 D$ stress field image around a representative indent $\left(F_{\text {max }}=\right.$ $3 \mathrm{~N}$ ) with $2 \mathrm{D}$ sliced images of the stress maps at $z=0,10,20,30$, and $40 \mu \mathrm{m}$. Only the compressive stress is pictured in the $3 \mathrm{D}$ representation for better visualization, and the scale bar is $20 \mu \mathrm{m}$. b Noise corrected lineic residual strain energy $E_{\text {res }}$ plotted against the depth; the area below the graph equals to the RSE. c log-log plot of IW and RSE against the indent-affected distance $d=3 h_{\max }$. $\mathbf{d}$ Residual energy ratio (RSE/IW) against the indent-affected distance $d$. A critical distance $d_{c}$ of about $20 \mu \mathrm{m}$ separates two regimes: for $d<d_{\mathrm{c}}$, RSE/IW increases linearly with $d$, whereas for $d>d_{\mathrm{c}}$, RSE/IW is constant. The black lines are guides for the eye. In both $\mathbf{c}$, $\mathbf{d}$ error bars stand for the standard deviations in $x$ and $y$ directions, and the blue crosses correspond to the data obtained with the aragonite sample.

maximum indentation depth $h_{\max }{ }^{54}$. Interestingly, while IW follows a power-law for increasing indentation depths as expected for contact type indentation tests ${ }^{55}$, RSE only shows a similar power-scaling for large enough indentation depths. This difference becomes most apparent when plotting the ratio RSE/IW (Fig. 3d). The ratio increases with increasing indentation depth up to $d \sim d_{\mathrm{c}}=20 \mu \mathrm{m}$, beyond which RSE/IW is constant. Otherwise said, beyond a critical indentation depth, nacre's microstructure stores a fixed portion of the externally supplied work (IW) into elastic energy (RSE), much akin to plastic hardening phenomena well known from plasticity ${ }^{56}$. In contrast, for $d<d_{c}=20 \mu \mathrm{m}$, this storage capacity is limited, which means that the externally supplied work is not stored into recoverable strain energy, but most likely dissipated through other toughening mechanisms at a constituent level such as crack deflection, sliding of platelets, mineral bridges, etc. $^{8,9,16}$. Indeed, to test this hypothesis, we carried out the same tests on monolithic aragonite crystals (blue cross-marks in Fig. 3c, d) and compared the results with those in nacre. Below the critical length, $d<d_{\mathrm{c}}=20 \mu \mathrm{m}$, the aragonite response follows a similar behavior as a nacre for both IW and RSE. For larger length scales, however, monolithic aragonite crystals dissipate energy through large crack formation, and therefore, aragonite crystals have significantly lower RSE (about two orders of magnitude lower than comparable indents on nacre). Furthermore, RSE/IW ratio of aragonite crystals is about $0.01-0.1 \%$ (Fig. 3d), explaining the absence of "strain-hardening" and the brittle behavior of aragonite. In summary, nacre behaves similarly to brittle aragonite in terms of energy storage when the length scale of the indent (crack) is smaller than $d_{c}$, but absorbs more energy when the indent size increases. These results hint at a strain-hardening mechanism in nacre, which develops in nacre's micro-texture at length scales greater than the critical length scale of $d_{c}=20 \mu \mathrm{m}$. The very existence of such a length scale implies a cooperative plastic deformation mechanism beyond the constituent level, which we aim to elucidate next.

Crystallographic texture of nacre and strain hardening. The brick-and-mortar microstructure of nacre is apparent in SEM images, but no distinct difference is found among the aragonite platelets (Fig. 4a-d). However, a significant difference appears in Raman images that separate aragonite platelets into two groups, based on the peak intensity ratio of the two peaks $L_{1}$ and $L_{3}$, denoted as $\mathrm{I}_{\mathrm{L} 1} / \mathrm{I}_{\mathrm{L} 3}$ (Fig. $4 \mathrm{e}-\mathrm{h}$, red and blue regions). The anisotropy in the intensity of Raman peaks has its origin in the preferential orientation of vibrating molecular units or crystal lattice vibrations with respect to the main axis of the sample and incident light polarization orientation ${ }^{57-59}$. In the case of nacre, the anisotropy originates from the fact that the incident laser is linearly polarized and $\mathrm{L}_{1}$ and $\mathrm{L}_{3}$ are two perpendicular lattice vibration modes ${ }^{51}$; therefore, the crystal orientation can be calculated from $\mathrm{I}_{\mathrm{L} 1} / \mathrm{I}_{\mathrm{L} 3}$. Indeed, Fig. $4 \mathrm{e}-\mathrm{h}$ show that the platelets are co-oriented along the growth direction (vertical direction in the images), creating stacks characterized by the same 

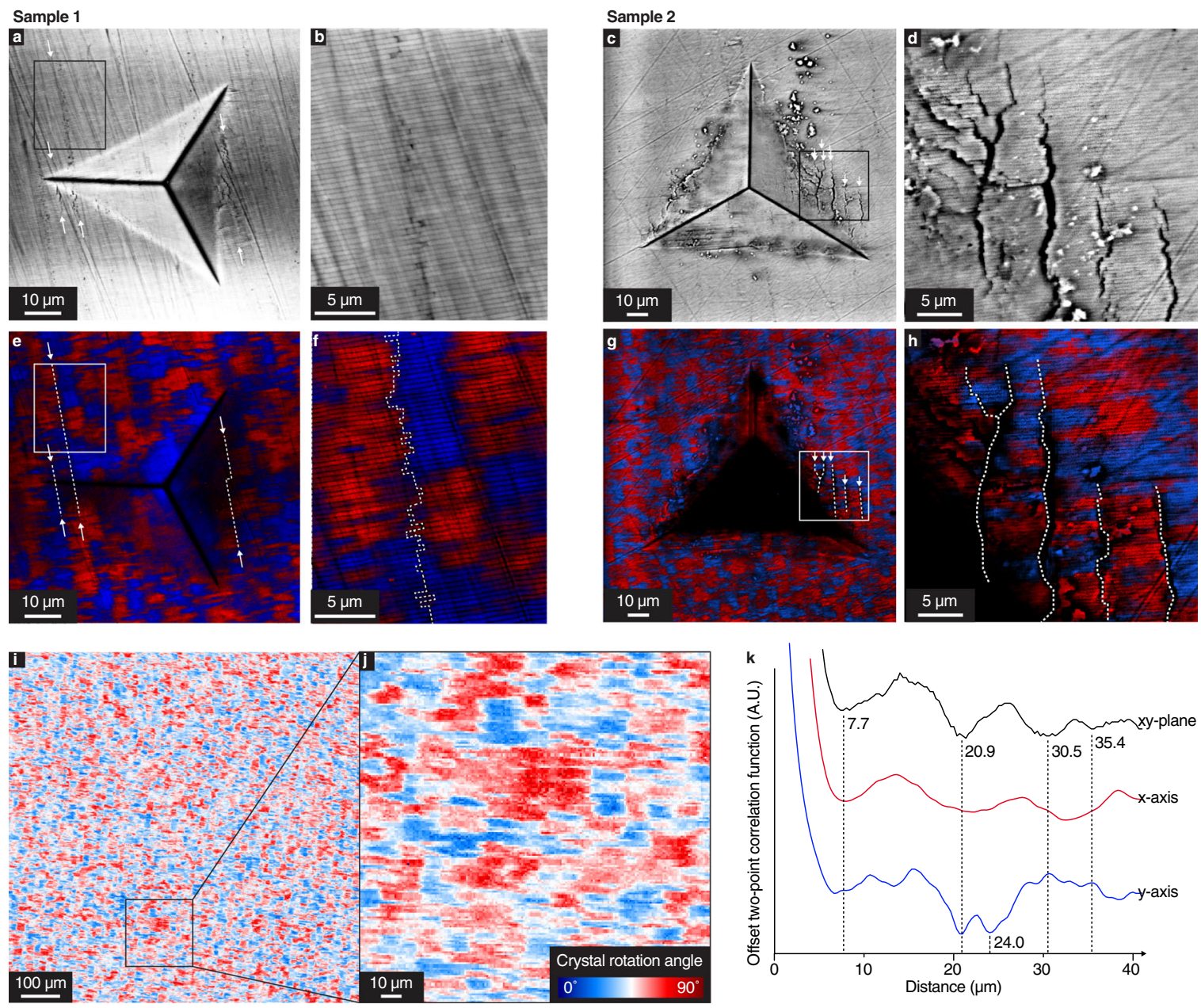

Fig. 4 Crack propagation and its relation to the aragonite stacks. a-d SEM images of two micro-indented nacre samples (Sample 1: $2.75 \mathrm{~N}$ and Sample 2: $10 \mathrm{~N}$ ); the white arrows indicate cracks emanating from the locus of the indent. e-h Raman component maps of the same regions as (a-d) with the cracks marked with arrows and dashed lines; the mapped area is divided into two red and blue components with low and high $L_{1} / L_{3}$ peak ratio, respectively. $\mathbf{i}, \mathbf{j}$ Crystal orientation map of aragonite platelets in a nacre sample; the crystal orientation is colored as indicated by the scale bar $\left(0-90^{\circ}\right)$. $\mathbf{k}$ Two-point correlation function computed in the $x y$-plane (black), $x$-direction (red), and $y$-direction (blue). The distances with local minimum are highlighted with dashed lines with the corresponding distances.

crystallographic orientation. Note that the crystallographic texture maps in Fig. 4e-h do not display a perfect checkerboard pattern since the length of the stacks is not uniform. For this reason, there exist parts where adjacent stacks have similar crystal orientation, and therefore, display the same color in Fig. 4e-h (e.g., bottom one-third of Fig. 4f).

Unlike natural platelet boundaries, cracks are visibly dilated in SEM images, and therefore, similarly to Wang et al. ${ }^{18}$, dilated boundaries (marked with arrows and dashed lines in Fig. 4a-h) were regarded as cracks in this study. Nevertheless, as previously discussed, it remains challenging to identify smaller cracks and crack tips, as the difference between such features and natural platelet boundaries becomes less discernable. The continuous straight streaks across the images are polishing marks, which are randomly oriented and independent from platelet direction unlike cracks and boundaries (see Supplementary Fig. 7). The cracks follow a complicated path near the indenter tip due to the high local stress generated by the indentation process; however, we can observe that the cracks eventually follow the inter-tablet boundaries as they propagate further from the indenter tips by overlaying the cracks on the SEM images and the Raman spectral maps. These co-oriented stacks have been observed previously in columnar nacre species using X-ray photoelectron emission spectromicroscopy (X-PEEM) $)^{10,46,47}$, transmission electron microscopy $(\mathrm{TEM})^{48}$, and electron backscatter diffraction $(\text { EBSD })^{49,50}$. However, to date, such a structure has not been linked to the mechanical behavior of nacre.

Raman spectroscopy evinces its advantages when correlating crystallographic texture and mechanical behavior. Indeed, information from both is obtained simultaneously from the peak properties: the crystallographic texture from the peak ratio of two perpendicular vibration mode peaks, and the mechanical behavior from the piezoRaman relationship. The crystal orientation (characterized by the Euler angles $\theta, \varphi, \psi$ defined in Supplementary Fig. 8) of an aragonite platelet is defined by the orientation of the crystal unit cell $(a, b, c)$ relative to the laboratory coordinate system (x, y, z). Aragonite platelets have a specific growth direction along the $\mathrm{c}$-axis ${ }^{14}$. Thus, by aligning the growth direction with the $y$-axis, we can define the crystal orientation with only one parameter, i.e., the crystal rotation angle $\psi$. The other Euler angles are $\theta=90^{\circ}, \varphi=0^{\circ}$. Raman intensity is $I \propto\left|e_{\mathrm{i}} \mathbf{R}_{\mathrm{xyz}} e_{\mathrm{s}}\right|^{2}$, where $\mathbf{R}_{\mathrm{xyz}}=\Phi_{\mathrm{xyz}} \mathbf{R} \Phi_{\mathrm{xyz}}^{\mathrm{T}}$ is the Raman tensor in the laboratory coordinate system, $\mathrm{R}$ is the Raman tensor of aragonite (Supplementary Table 1), and $\boldsymbol{\Phi}_{\mathrm{xyz}}$ is Euler's matrix with $(\theta, \varphi, \psi)=(90,0, \psi)$. Inputting the incident laser polarization $e_{\mathrm{i}}=$ $(1,0,0)$ to the equations, the peak intensity ratio of $L_{1}$ and $L_{3}$ peaks 
takes the following form:

$$
\mathrm{I}_{\mathrm{L} 1} / \mathrm{I}_{\mathrm{L} 3} \propto \frac{\mathrm{d}^{2} \sin ^{2} \psi}{\mathrm{e}^{2} \cos ^{2} \psi} \propto \tan ^{2} \psi
$$

Hence, $\psi$ is expressed as a function of the peak intensity ratio $\mathrm{I}_{\mathrm{L} 1} / \mathrm{I}_{\mathrm{L} 3}$. The crystal orientation map of a broader region of the nacre sample, plotted using the crystal rotation angle $(\psi)$, is shown in Fig. 4i, j. The blue and red colors of Fig. 4i, j correspond to $0^{\circ}$ and $90^{\circ}$ of crystal rotation, meaning that the crystal's a-axis is parallel and perpendicular to the laser polarization, respectively. Note that the color of the interface appears to be white $\left(45^{\circ}\right)$ due to limitations in spatial resolution; the spectrum of the interface is a weighted sum of the spectra of adjacent platelets. The periodicity of the crystallographically co-oriented stacks of aragonite platelets ranging between $10 \mu \mathrm{m}$ and $100 \mu \mathrm{m}$ in length, is clearly visible (see also Fig. $4 \mathrm{j}$ for details on a smaller region).

Using these orientation maps, we analyzed the periodicity of the crystal orientation by using the two-point correlation function $S_{2}^{(i)}(r)$, defined as the probability of both ends of a randomly placed segment (length $=r$ ) to be in the same phase $i^{60}$. The two phases can be interchanged without loss of generality when analyzing the crystallographic texture of nacre. Therefore, we compute the sum $S(r) \equiv S_{2}^{(1)}(r)+S_{2}^{(2)}(r)$ (Supplementary Fig. 9a). In the case of the existence of a characteristic length $\lambda$ in the map, $S(n \lambda)$ would be close to local minima, when $n=1,3,5 \ldots 60,61$. The local minima found in $S(r)$ in the $x y$-plane (black line in Fig. 4k) indicate the characteristic lengths 7.7, 20.9, 30.5, and 35.4 $\mu \mathrm{m}$ (see Supplementary Fig. 9b for the larger distances). Since the material has a directionality along the $x$ - and $y$-direction, $S(r)$ was calculated in these two directions (red and blue lines). The characteristic length in the $x$-direction is $\lambda_{\mathrm{x}} \sim 7 \mu \mathrm{m}$, corresponding to the width of the aragonite platelets $(\sim 8 \mu \mathrm{m})^{9}$. In the $y$ direction, $S(r)$ exhibits two minima, which indicates that the aragonite stacks have a characteristic length $\lambda_{\mathrm{y}} \sim 21-24 \mu \mathrm{m}$, which approximates the critical length of $\sim 20 \mu \mathrm{m}$ that we found through RSE (Fig. 3d).

These results offer strong evidence that stacks of crystallographically co-oriented aragonite platelets contribute to the exceptional mechanical properties of nacre. First, indentationinduced cracks propagate along the aragonite stack boundaries, suggesting that each aragonite stack acts as a cooperative unit, as opposed to the previous focus on the individual movement of aragonite platelets ${ }^{18,19}$. Second, the nacre possesses an effective strain hardening mechanism that is gradually activated as the indentation depth increases up to a critical length $(\sim 20 \mu \mathrm{m})$ set by the size of the aragonite stacks. The co-oriented aragonite stacks, similarly to supertablets ${ }^{21}$, highlight a toughening mechanism operating at the length scale that is beyond the brick-and-mortar structure characteristic for nacre.

In order to support micro-indentation and piezo-Raman results, we performed macroscopic tests on micro-notched nacre samples. First, the crystal orientation of beams of columnar nacre loaded in 3-point bending and sheet nacre that has been tested without a notch is analyzed using Polarization-dependent Imaging Contrast (PIC) maps (Fig. 5a-d), where the gray level quantitatively represents the orientation of the aragonite crystal. The co-oriented stacks of aragonite platelets are clearly observed in columnar nacre (Fig. 5a, c). Even though sheet nacre does not have a columnar microstructure, one can observe stacks of cooriented platelets forming stair-shaped mesostructures (Fig. 5b, d). The cracks were observed at the interface of the co-oriented platelet stacks (Fig. 5c, d), showing that both columnar and sheet nacre display a similar response. The differences in the mechanical contribution of the platelet stacks in columnar and sheet nacre will be a subject of future work.
In columnar nacre, the cracks propagate along the stack boundaries as seen in the PIC maps (Fig. 5e, f). The crack initially propagated through the platelets near the notch for about 10 platelets. Then, the crack diverts to a stack boundary and propagates in mixed mode I + II following the path of lowest crack propagation resistance. When the crack driving force in the mode I plane becomes dominant, the crack jumps to the adjacent stack boundary and restarts the propagation process along the boundary (Fig. 5f). The crack jump is observed where the crystal orientation changes (dashed line in Fig. 5f), supporting the mechanical contribution of the co-oriented stacks. Also, see Supplementary Fig. 10 for a high-resolution SEM image of crack jumps in a columnar nacre sample.

The gray level in each pixel of the PIC map corresponds to the orientation of the aragonite crystal $c$-axis. The orientation (contrast) of platelets left and right to the crack in Fig. $5 \mathrm{e}$ is plotted in Fig. $5 \mathrm{~g}$, where red and yellow data points indicate whether or not the crystal orientation on the left $\left(\theta_{\mathrm{L}}\right)$ and right $\left(\theta_{\mathrm{R}}\right)$ of the crack are statistically significantly different. The majority of cracks $(226 / 297$, or $76 \%)$ propagated between misoriented platelets, and $71 / 297$, or $24 \%$, were between cooriented tablets. Since the gray level is homogeneous within each platelet in the PIC images, the red points indicate that the crack propagated along the stack boundaries. This crack propagation pattern clearly demonstrates the mechanical contribution of cooriented stacks in nacre's toughening mechanism.

Conclusion. In this study, mechanical results from microindentation, 3-point bending, and in-situ compression tests were compared with the chemical, structural, and mechanical properties derived from Raman spectroscopy. Correlating the crystallographic texture and the residual strain energy revealed that energy is effectively stored in the aragonite stacks when the indent is larger than the stack's characteristic size. This finding indicates that the collective movement of the co-oriented aragonite stacks contributes to the strain hardening of the nacre. As such, aragonite stacks define another hierarchical level that contributes to the outstanding mechanical performance of nacre. This work also highlights the effectiveness of correlative SEM-Raman imaging tools in quantifying and mapping the plastic deformation and the residual stress field, as well as analyzing the crystallographic texture with sub-micron spatial resolution.

\section{Methods}

Sample preparation for indentation and Raman spectroscopy. Dry nacre samples were cut from an abalone shell (Haliotis rufescens) using a diamond saw (see Supplementary Note 2 regarding the sample humidity condition). For the in-situ piezo-Raman compression test, a rectangular block of nacre $(0.8 \times$ $\left.1.0 \times 1.9 \mathrm{~mm}^{3}\right)$ was prepared with the growth direction (c-axis) oriented along the longest dimension of the sample. For the micro-indentation tests, nacre samples $\left(\sim 20 \times 5 \times 5 \mathrm{~mm}^{3}\right)$ were embedded in epoxy resin with the cross-section exposed to the air before being manually polished in dry conditions with a series of $\mathrm{SiC}$ papers of decreasing surface roughness to reach a surface roughness of a few hundred nanometers. Monolithic aragonite samples $\left(\sim 5 \times 5 \times 5 \mathrm{~mm}^{3}\right)$ were obtained from an aragonite cluster. They were embedded in resin with the c-axis parallel to the surface and polished similarly to nacre samples.

Micro-indentation. Indentation tests were carried out using a three-sided, pyramid-like Berkovich indenter mounted on a Micro Combi Tester (Anton Paar, Graz, Austria). Each indentation test consists of measuring the indentation depth of the indenter resulting from a symmetric load profile composed of an increasing ramp of load performed at $14 \mathrm{~N} \mathrm{~min}^{-1}$ up to a constant value $F_{\max }$ maintained for $10 \mathrm{~s}$ and followed by a decreasing ramp of load with identical parameters. The maximum load $F_{\max }$ was varied between $10 \mathrm{mN}$ and $30 \mathrm{~N}$. Finally, for each indent, the local values of the hardness and the indentation modulus were determined by the method of Oliver and Pharr ${ }^{53}$.

SEM, Raman imaging and analysis. Correlative SEM and Raman images were produced using the RISE microscopy system (WITec, Ulm, Germany and Tescan, 

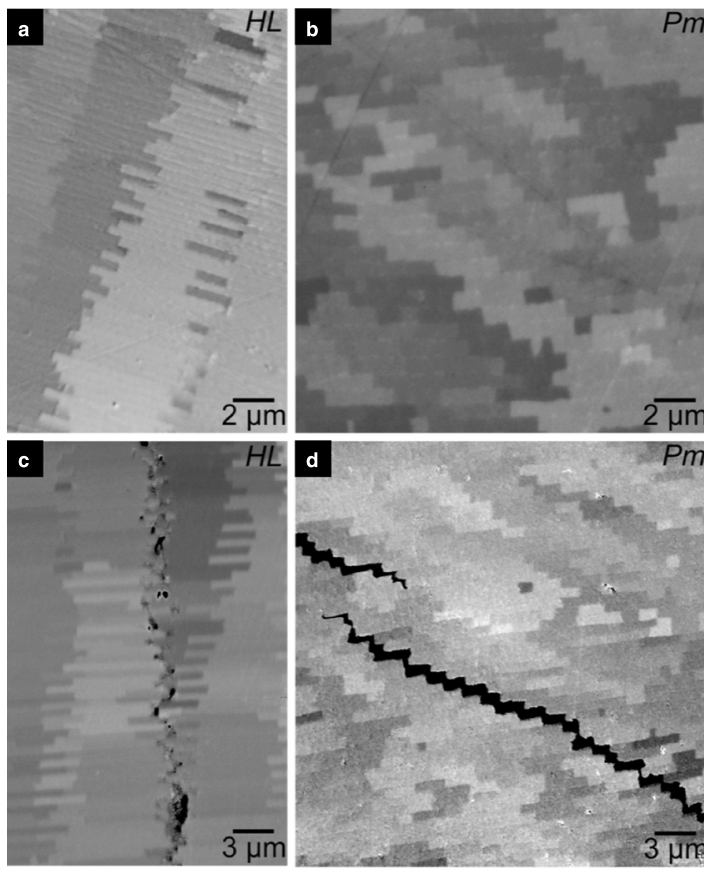

$-90^{\circ}$

$0^{\circ}$
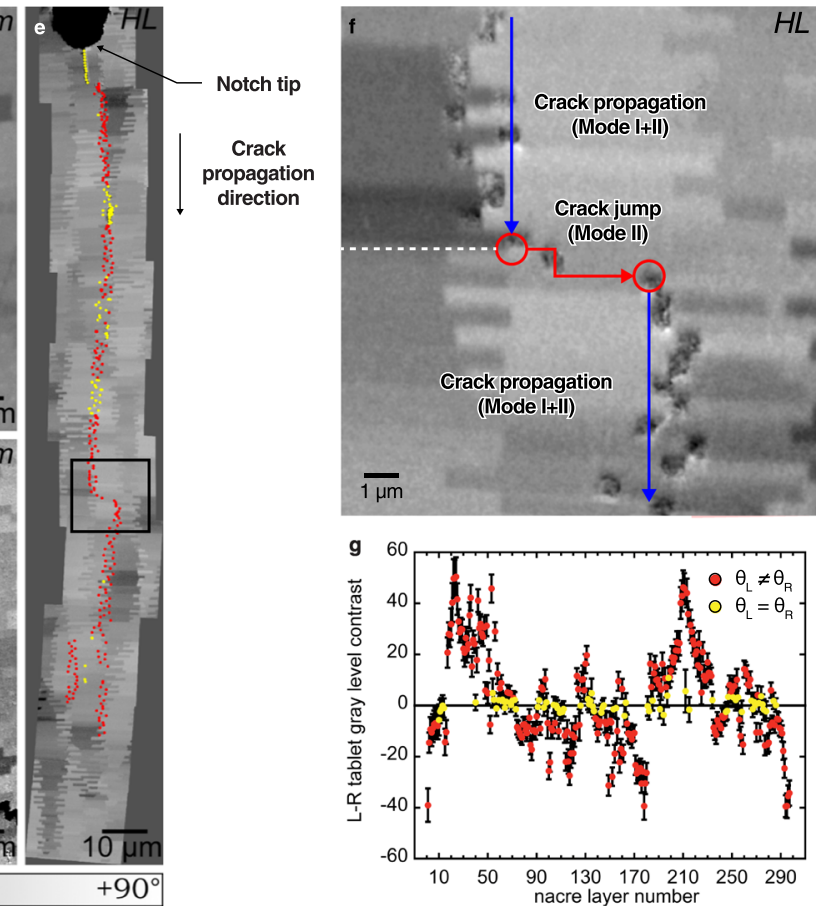

Fig. 5 The cracks propagate along co-oriented stacks in both columnar and sheet nacre. PIC maps of a columnar nacre (Haliotis laevigata) and $\mathbf{b}$ sheet nacre (Pinctada margaritifera). PIC maps of cracks propagating through c columnar nacre and $\mathbf{d}$ sheet nacre. e PIC map showing the crystal orientation of each tablet in the columnar nacre sample. $\mathbf{f}$ Magnified PIC map of the boxed region in (e). The crack propagation (mode I + II) on the stack boundaries and the crack jump (mode II) from the stack boundary to the next boundary are observed. $\mathbf{g}$ The orientation of the $c$-axis of the aragonite crystal platelet on the left $\left(\theta_{L}\right)$ or right $\left(\theta_{R}\right)$ of the crack, with respect to vertical in the image plane. The red and yellow data points indicate whether $\theta_{L}$ and $\theta_{R}$ are statistically significantly different or not. The grayscale in a-f indicates the $c$-axis orientation with respect to the vertical.

Brno, Czech Republic) and a confocal Raman system (WITec). Raman maps were acquired using a continuous $532 \mathrm{~nm}$ laser beam with an accumulation time of $0.1-0.15 \mathrm{~s}$ per point. The Raman map sizes were determined to include at least 20 times of the indent height in radius from the indent core. Different spatial resolutions $(0.25-2 \mu \mathrm{m})$ were used for scans of different sizes. Stack scans, each series of 2-dimensional scans with different focal lengths, were performed on multiple indents to analyze the material in $3 \mathrm{D}$. The scan volume depended on the size of the indents, and the scan resolution was $4 \mu \mathrm{m}$ per pixel for lower resolution scans and $2 \mu \mathrm{m}$ per pixel for higher resolution scans. The data were collected with a CCD behind a $600 \mathrm{~g} \mathrm{~mm}^{-1}$ grated UHTS Raman Spectrometer (WITec).

WITec Project Five, MATLAB, Origin Pro 9, and ImageJ ${ }^{62}$ software were used for image processing and analyses. Cosmic ray removal and background subtraction were performed to increase the signal-to-noise ratio. The $\mathrm{L}_{1}, \mathrm{~L}_{3}$, and $v_{1}$ peaks were fitted with three Lorentzian functions, and the peak position, width, and intensity were extracted from the fit for further analyses. A median filter was applied to exclude outlier data points. Residual stress occurs naturally due to the structural mismatch of aragonite platelets and organic matrices ${ }^{63}$. To subtract the natural residual stress, the $v_{0}$ value was calibrated for each indent using the median peak position of the nacre in native condition.

Piezo-Raman. A load cell (Interface, Scottsdale, Arizona, USA) with the maximum load capacity of $200 \mathrm{~N}$ was used for measuring the applied force, and the average stress was calculated by dividing the applied force by the cross-section area of the sample $\left(0.8 \times 1.0 \mathrm{~mm}^{2}\right)$. The nacre block was subjected to a compressive load perpendicular to the platelets. At each stress level, Raman spectra of 100 points were obtained from an area of $10 \times 10 \mu \mathrm{m}^{2}$ at the center of the nacre sample surface. Each Raman spectrum was fitted with three Lorentzian functions, each corresponding to $\mathrm{L}_{1}, \mathrm{~L}_{3}$, and $v_{1}$ peaks. The average and standard deviation of the Raman peak position were determined, and the average peak shifts were fitted to a linear function.

Macroscopic mechanical tests. The Haliotis laevigata nacre samples were polished into beams with size $2 \mathrm{~mm} \times 2 \mathrm{~mm} \times 10 \mathrm{~mm}$ using SiC paper to a $1-\mu \mathrm{m}$ surface finish using water for grinding and water-based diamond suspension for the final polishing steps. Notching was done with a water-irrigated low-speed saw, and then the saw-cut notch was sharpened to a crack tip radius of roughly $5-10 \mu \mathrm{m}$ by polishing the root of the notch with a razor blade irrigated with $1-\mu \mathrm{m}$ diamond solution; the total depth of the notch was about half of the sample thickness. Samples were kept in salt water for at least $12 \mathrm{~h}$ prior to testing. Bending test was done with a Gatan Microtest $2 \mathrm{kN}$ bending stage (Gatan, Abington, UK) using a loading span of $6 \mathrm{~mm}$ in a S-4300SE/N variable pressure scanning electron microscope (Hitachi America, Pleasanton, CA, USA), allowing continuous observation of the crack length on the sample's surface throughout mechanical testing. Care was taken to ensure that the sample did not break completely, but was approximately $1 / 2$ cracked, so PEEM analysis could be done at the crack locations.

X-PEEM analysis and PIC mapping. After the 3-point bending and cracking, the Haliotis laevigata nacre sample was embedded into EpoFix (EMS, Hatfield, PA, USA) and polished down to $50-\mathrm{nm} \mathrm{Al}_{2} \mathrm{O}_{3}$ suspension in water (MasterPrep, Buehler, Lake Bluff, IL, USA). It was then coated with differential-thickness (40 $\mathrm{nm}, 1 \mathrm{~nm} \mathrm{Pt}$ ) as described before ${ }^{64,65}$, and analyzed with PEEM in precisely the same location where the crack was induced by 3-point bending. The other samples of Haliotis laevigata and Pinctada margaritifera were embedded, polished, and coated, similarly.

$\mathrm{X}$-ray photoelectron emission spectromicroscopy (X-PEEM) was performed using the PEEM-3 microscope on beamline 11.0.1 at the Advanced Light Source in Berkeley, CA. The elliptically polarizing undulator (EPU) at this beamline was calibrated to provide precise linear X-ray polarization and reproducible intensities at polarization angles between $0^{\circ}-90^{\circ}$ with a $5^{\circ}$ step size ( 19 images per dataset). All PEEM datasets were acquired with a $20-\mu \mathrm{m}$ field of view, in duplicate with reproducible results. For the long crack in Fig. 5e, ten partly overlapping datasets were acquired.

For each 19-image dataset, we calculated the orientation of the c-axis in three dimensions and displayed it semi-quantitatively as gray level in Fig. 5a-e, where the middle gray level corresponds to a vertical c-axis, one at $+45^{\circ}$ points to the top right (white) and one at $-45^{\circ}$ points to the top left (black) according to the grayscale shown in Fig. 5e. Since nacre tablets always only have $\pm 30^{\circ}$ from the vertical in both columnar and sheet nacre ${ }^{47,65,66}$, only intermediate gray levels are visible, and no black or white tablets appear. In the end, and for publication and display only, not for the angle measurements in Fig. 5g, the contrast was enhanced in Adobe Photoshop using the "level" function. For all of the images, the sample voltage was $-15 \mathrm{kV}$, and the photon energy was kept constant at $290.3 \mathrm{eV}$, the carbon $\mathrm{K}$ edge $\pi^{*}$ peak $^{46}$, which is strongly polarization-sensitive in all carbonates ${ }^{67}$. Fitting the polarization spectrum to the curve $\mathrm{y}=\mathrm{A}+\mathrm{B} \cos ^{2}\left(\mathrm{EPU}^{\circ}+\mathrm{C}\right)$, with the Levenberg-Marquardt least-squares analysis method, yields accurate identification of the position of the curve maximum and thereby determination of c-axis orientation. Performing this fit for every pixel in the stack of images obtained from PEEM-3, and composing the results into a single grayscale image yields a Polarization-dependent Imaging Contrast (PIC) map, in which the in-plane c-axis orientation angle is semi-quantitatively represented by a gray level between $0-255$. 
These gray levels correspond to the possible $180^{\circ}$-range of orientations between $-90^{\circ}$ and $+90^{\circ}$, with $0^{\circ}$ corresponding to a vertical in-plane $c$-axis.

Radiation damage was undetectable in repeat acquisitions, as the mineral component of biominerals is not radiation sensitive ${ }^{68}$

\section{Data availability}

The data that support the findings of this study are available from the corresponding author upon reasonable request.

Received: 17 February 2020; Accepted: 20 September 2020;

Published online: 28 October 2020

\section{References}

1. Fratzl, P. \& Weinkamer, R. Nature's hierarchical materials. Prog. Mater. Sci. 52, 1263-1334 (2007).

2. Meyers, M. A., Chen, P.-Y., Lin, A. Y.-M. \& Seki, Y. Biological materials: structure and mechanical properties. Prog. Mater. Sci. 53, 1-206 (2008)

3. Bonderer, L. J., Studart, A. R. \& Gauckler, L. J. Bioinspired design and assembly of platelet reinforced polymer films. Science 319, 1069-1073 (2008).

4. Wegst, U. G. K., Bai, H., Saiz, E., Tomsia, A. P. \& Ritchie, R. O. Bioinspired structural materials. Nat. Mater. 14, 23-36 (2015).

5. Dunlop, J. W. C., Weinkamer, R. \& Fratzl, P. Artful interfaces within biological materials. Mater. Today 14, 70-78 (2011).

6. Currey, J. D. Mechanical properties of mother of pearl in tension. Proc. R. Soc. London Biol. Sci. 196, 443-463 (1977).

7. Jackson, A. P., Vincent, J. F. V. \& Turner, R. M. The mechanical design of nacre. Proc. R. Soc. London Biol. Sci. 234, 415-440 (1988).

8. Sarikaya, M., Gunnison, K. E., Yasrebi, M. \& Aksay, I. A. Mechanical Property-Microstructural Relationships in Abalone Shell. MRS Proc. 174, 109-116 (1989).

9. Meyers, M. A. \& Chen, P.-Y. Biological Materials Science (Cambridge University Press, 2014)

10. Olson, I. C., Kozdon, R., Valley, J. W. \& Gilbert, P. U. P. A. Mollusk shell nacre ultrastructure correlates with environmental temperature and pressure. J. Am. Chem. Soc. 134, 7351-7358 (2012).

11. Gao, R., Wang, R., Feng, X. \& Zhang, G. Growth of nacre biocrystals by selfassembly of aragonite nanoparticles with novel subhedral morphology. Crystals 10, 11-14 (2020).

12. Liu, J., Sarikaya, M. \& Aksay, I. A. A hierarchically structured model composite: a TEM study of the hard tissue of red abalone. Mat. Res. Soc. Symp. Proc. 255, 9-17 (1992)

13. Nassif, N. et al. Amorphous layer around aragonite platelets in nacre. Proc. Natl Acad. Sci. USA 102, 12653-12655 (2005).

14. Lin, A. \& Meyers, M. A. Growth and structure in abalone shell. Mater. Sci. Eng. A 390, 27-41 (2005).

15. Smith, B. L. et al. Molecular mechanistic origin of the toughness of natural adhesives, fibres and composites. Nature 399, 761-763 (1999).

16. Meyers, M. A., Lin, A. Y.-M., Chen, P.-Y. \& Muyco, J. Mechanical strength of abalone nacre: Role of the soft organic layer. J. Mech. Behav. Biomed. Mater. 1, 76-85 (2008).

17. Evans, A. G. et al. Model for the robust mechanical behavior of nacre. J. Mater. Res. 16, 2475-2484 (2001).

18. Wang, R. Z., Suo, Z., Evans, A. G., Yao, N. \& Aksay, I. A. Deformation mechanisms in nacre. J. Mater. Res. 16, 2485-2493 (2001).

19. Barthelat, F., Li, C.-M., Comi, C. \& Espinosa, H. D. Mechanical properties of nacre constituents and their impact on mechanical performance. J. Mater. Res. 21, 1977-1986 (2006).

20. Askarinejad, S. \& Rahbar, N. Toughening mechanisms in bioinspired multilayered materials. J. R. Soc. Interface 12, 20140855 (2015).

21. Wang, R. \& Gupta, H. S. Deformation and fracture mechanisms of bone and nacre. Annu. Rev. Mater. Res. 41, 41-73 (2011).

22. Song, F. \& Bai, Y. L. Effects of nanostructures on the fracture strength of the interfaces in nacre. J. Mater. Res. 18, 1741-1744 (2003).

23. Prime, M. B. Cross-sectional mapping of residual stresses by measuring the surface contour after a cut. J. Eng. Mater. Technol. 123, 162-168 (2001).

24. Pan, B., Qian, K., Xie, H. \& Asundi, A. Two-dimensional digital image correlation for in-plane displacement and strain measurement: a review. Meas. Sci. Technol. 20, 062001 (2009).

25. Steiner, M. A. et al. Path length dependent neutron diffraction peak shifts observed during residual strain measurements in U-8 wt\% Mo castings. J. Appl. Crystallogr. 50, 851-858 (2017).

26. Fitzpatrick, M. E. \& Lodini, A. Analysis of Residual Stress by Diffraction using Neutron and Synchrotron Radiation (Taylor \& Francis, 2003)
27. Poulsen, H. F. et al. Three-dimensional maps of grain boundaries and the stress state of individual grains in polycrystals and powders. J. Appl. Crystallogr. 34, 751-756 (2001).

28. Vaudin, M. D., Gerbig, Y. B., Stranick, S. J. \& Cook, R. F. Comparison of nanoscale measurements of strain and stress using electron back scattered diffraction and confocal Raman microscopy. Appl. Phys. Lett. 93, 1-3 (2008).

29. Toporski, J., Dieing, T. \& Hollricher, O. Confocal Raman Microscopy (Springer International Publishing AG, 2018).

30. Jiroušek, O., Jandejsek, I. \& Vavř́k, D. Evaluation of strain field in microstructures using micro-CT and digital volume correlation. J. Instrum. 6 C01039 (2011).

31. Barthelat, F. \& Espinosa, H. D. An experimental investigation of deformation and fracture of nacre-mother of pearl. Exp. Mech. 47, 311-324 (2007).

32. Masic, A. \& Weaver, J. C. Large area sub-micron chemical imaging of magnesium in sea urchin teeth. J. Struct. Biol. 189, 269-275 (2015).

33. Amini, S. et al. Textured fluorapatite bonded to calcium sulphate strengthen stomatopod raptorial appendages. Nat. Commun. 5, 3187 (2014).

34. Gentleman, E. et al. Comparative materials differences revealed in engineered bone as a function of cell-specific differentiation. Nat. Mater. 8, 763-770 (2009).

35. Pezzotti, G. \& Sakakura, S. Study of the toughening mechanisms in bone and biomimetic hydroxyapatite materials using Raman microprobe spectroscopy. J. Biomed. Mater. Res. - Part A 65, 229-236 (2003).

36. Pezzotti, G. Raman piezo-spectroscopic analysis of natural and synthetic biomaterials. Anal. Bioanal. Chem. 381, 577-590 (2005)

37. Schrof, S., Varga, P., Galvis, L., Raum, K. \& Masic, A. 3D Raman mapping of the collagen fibril orientation in human osteonal lamellae. J. Struct. Biol. 187, 266-275 (2014)

38. Amini, S., Tadayon, M., Idapalapati, S. \& Miserez, A. The role of quasiplasticity in the extreme contact damage tolerance of the stomatopod dactyl club. Nat. Mater. 14, 943-950 (2015).

39. Ganesan, S., Maradudin, A. A. \& Oitmaa, J. A Lattice Type of Morphic Effects in Crystals of the Diamond structure. Ann. Phys. 56, 556-594 (1970).

40. Anastassakis, E., Pinczuk, A., Burstein, E., Pollak, F. H. \& Cardona, M. Effect of static uniaxial stress on the Raman spectrum of silicon. Solid State Commun 8, 133-138 (1970)

41. De Wolf, I. Micro-Raman spectroscopy to study local mechanical stress in silicon integrated circuits. Semicond. Sci. Technol. 11, 139-154 (1996).

42. Animoto, S. T., Chang, D. J. \& Birkitt, A. D. Stress measurement in MEMS using Raman spectroscopy. SPIE Proc. 3512, 123-129 (1998).

43. Bissett, M. A., Tsuji, M. \& Ago, H. Strain engineering the properties of graphene and other two-dimensional crystals. Phys. Chem. Chem. Phys. 16, 11124-11138 (2014)

44. Dukino, R. D. \& Swain, M. V. Comparative measurement of indentation fracture toughness with Berkovich and Vickers indenters. J. Am. Ceram. Soc. 75, 3299-3304 (1992)

45. Broitman, E. Indentation hardness measurements at macro-, micro-, and nanoscale: a critical overview. Tribol. Lett. 65, 1-18 (2017).

46. Metzler, R. A. et al. Architecture of columnar nacre, and implications for its formation mechanism. Phys. Rev. Lett. 98, 1-4 (2007).

47. Gilbert, P. U. P. A. et al. Gradual ordering in red abalone nacre. J. Am. Chem Soc. 130, 17519-17527 (2008).

48. Mukai, H., Saruwatari, K., Nagasawa, H. \& Kogure, T. Aragonite twinning in gastropod nacre. J. Cryst. Growth 312, 3014-3019 (2010).

49. Griesshaber, E. et al. Homoepitaxial meso- and microscale crystal coorientation and organic matrix network structure in Mytilus edulis nacre and calcite. Acta Biomater 9, 9492-9502 (2013).

50. Gopalan, H. \& Chokshi, A. H. The mechanical behavior of nacre across length scales. J. Mech. Behav. Biomed. Mater. 78, 96-107 (2018).

51. De La Pierre, M. et al. The Raman spectrum of $\mathrm{CaCO}_{3}$ polymorphs calcite and aragonite: a combined experimental and computational study. J. Chem. Phys. 140, 164509 (2014)

52. Johnson, K. L. Contact Mechanics (Cambridge University Press, 1985).

53. Oliver, W. C. \& Pharr, G. M. Measurement of hardness and elastic modulus by instrumented indentation: advances in understanding and refinements to methodology. J. Mater. Res. 19, 3-20 (2004)

54. Ulm, F.-J. et al. Does microstructure matter for statistical nanoindentation techniques? Cem. Concr. Compos. 32, 92-99 (2010).

55. Borodich, F. M. The hertz frictional contact between nonlinear elastic anisotropic bodies (the similarity approach). Int. J. Solids Struct. $\mathbf{3 0}$ 1513-1526 (1993)

56. Fleck, N. A., Muller, G. M., Ashby, M. F. \& Hutchinson, J. W. Strain gradient plasticity: Theory and experiment. Acta Metall. Mater. 42, 475-487 (1994).

57. Masic, A. et al. Observations of multiscale, stress-induced changes of collagen orientation in tendon by polarized Raman spectroscopy. Biomacromolecules 12, 3989-3996 (2011) 
58. Bower, D. I. Investigation of molecular orientation distributions by polarized Raman scattering and polarized fluorescence. J. Polym. Sci. Part A-2 Polym. Phys. 10, 2135-2153 (1972).

59. Sourisseau, C. Polarization measurements in macro- and micro-Raman spectroscopies: molecular orientations in thin films and azo-dye containing polymer systems. Chem. Rev. 104, 3851-3891 (2004).

60. Torquato, S. Random Heterogeneous Materials: Microstructure and Macroscopic Properties (Springer, New York, NY, 2002).

61. Carolan, D., Chong, H. M., Ivankovic, A., Kinloch, A. J. \& Taylor, A. C. Cocontinuous polymer systems: a numerical investigation. Comput. Mater. Sci. 98, 24-33 (2015).

62. Schindelin, J. et al. Fiji: An open-source platform for biological-image analysis. Nat. Methods 9, 676-682 (2012).

63. Seknazi, E. \& Pokroy, B. Residual strain and stress in biocrystals. Adv. Mater. 30, 1-6 (2018).

64. De Stasio, G., Frazer, B. H., Gilbert, B., Richter, K. L. \& Valley, J. W. Compensation of charging in X-PEEM: a successful test on mineral inclusions in $4.4 \mathrm{Ga}$ old zircon. Ultramicroscopy 98, 57-62 (2003).

65. Olson, I. C. et al. Crystal lattice tilting in prismatic calcite. J. Struct. Biol. 183, 180-190 (2013).

66. Gilbert, P. U. P. A. et al. Nacre tablet thickness records formation temperature in modern and fossil shells. Earth Planet. Sci. Lett. 460, 281-292 (2017).

67. Gilbert, P. U. P. A., Young, A. \& Coppersmith, S. N. Measurement of c-axis angular orientation in calcite $\left(\mathrm{CaCO}_{3}\right)$ nanocrystals using X-ray absorption spectroscopy. Proc. Natl Acad. Sci. USA 108, 11350-11355 (2011).

68. Parasassi, T., Sapora, O., Giusti, A. M., De Stasio, G. \& Ravagnan, G. Alterations in erythrocyte membrane lipids induced by low doses of ionizing radiation as revealed by 1,6-diphenyl-1,3,5-hexatriene fluorescence lifetime. Int. J. Radiat. Biol. 59, 59-69 (1991).

\section{Acknowledgements}

This work was funded by the scholarship program of the Kwanjeong Educational Foundation. The authors thank Andre Studart, Tommaso Magrini, and Clara Minas from ETH Zurich for fruitful discussions. PUPAG acknowledges $20 \%$ support from the National Science Foundation (grant BMAT-1105167) and 60\% support from the Department of Energy (grant DOE-BES-CSGB-Geosciences DE-FG02-07ER15899). The PEEM experiments were done at the Advanced Light Source (ALS), which is supported by the Director, Office of Science, Office of Basic Energy Sciences, US Department of Energy under Contract No. DE-AC02-05CH11231.

\section{Author contributions}

A.M. conceived the idea and designed the study. H.-C.L. and T.D. prepared the samples H.-C.L. performed the SEM and Raman spectroscopy experiments, processed the data, and interpreted the results. B.G. and R.O.R. performed and interpreted the 3-point bending experiments. P.U.P.A.G. conceived the nacre cracking experiment, provided the samples for 3-point bending, performed Polarization-dependent Imaging Contrast mapping, processed the data, and interpreted the results. T.D. performed microindentation experiments, and T.D. and F.-J.U. interpreted the results. H.-C. L. and A.M. drafted the paper with input and edits from all other authors.

\section{Competing interests}

The authors declare no competing interests.

\section{Additional information}

Supplementary information is available for this paper at https://doi.org/10.1038/s43246020-00078-y.

Correspondence and requests for materials should be addressed to A.M.

Reprints and permission information is available at http://www.nature.com/reprints

Publisher's note Springer Nature remains neutral with regard to jurisdictional claims in published maps and institutional affiliations.

(c) (i) Open Access This article is licensed under a Creative Commons Attribution 4.0 International License, which permits use, sharing, adaptation, distribution and reproduction in any medium or format, as long as you give appropriate credit to the original author(s) and the source, provide a link to the Creative Commons license, and indicate if changes were made. The images or other third party material in this article are included in the article's Creative Commons license, unless indicated otherwise in a credit line to the material. If material is not included in the article's Creative Commons license and your intended use is not permitted by statutory regulation or exceeds the permitted use, you will need to obtain permission directly from the copyright holder. To view a copy of this license, visit http://creativecommons.org/ licenses/by/4.0/.

(C) The Author(s) 2020 